\title{
AN APPLICATION OF HOLMGREN'S THEOREM AND CONVEXITY WITH RESPECT TO DIFFERENTIAL OPERATORS WITH FLAT CHARACTERISTIC CONES $\left(^{1}\right)$
}

BY

\section{E. C. ZACHMANOGLOU}

1. Introduction. We consider linear partial differential operators of order $m$ in $n$-dimensional space

$$
P(x, D)=\sum_{|\alpha| \leqq m} a^{\alpha}(x) D^{\alpha},
$$

where $x=\left(x_{1}, \ldots, x_{n}\right)$ is a point in $R_{n}, \alpha=\left(\alpha_{1}, \ldots, \alpha_{n}\right)$ is an $n$-tuple of nonnegative integers with $|\alpha|=\sum_{i=1}^{n} \alpha_{j}$ and $D^{\alpha}=D_{1}^{\alpha_{1}} \cdots D_{n}^{\alpha_{n}}$ with $D_{j}=\partial / \partial x_{j}$. The principal part $P_{m}(x, D)$ is the homogeneous part of order $m$,

$$
P_{m}(x, D)=\sum_{|\alpha|=m} a^{\alpha}(x) D^{\alpha} .
$$

At a fixed point $x^{0} \in R_{n}$ the (real) zeroes of $P_{m}\left(x^{0}, \xi\right)$ form a cone in $R_{n}$ which is called the (real) characteristic cone of $P(x, D)$ at $x^{0}$.

In this paper we study only differential operators with flat characteristic cones. Specifically we assume that at each point of an open set $\Omega$ the characteristic cone of $P(x, D)$ is contained in the orthogonal complement of a fixed subspace $W$ of $R_{n}$ different from $\{0\}$. In $\S 2$, by a repeated application of Holmgren's theorem (as extended to distribution solutions by Hormander [1]), we show that if $P(x, D)$ has analytic coefficients in $\Omega$ and if $u$ is a distribution solution of $P(x, D) u=0$ in $\Omega$ which vanishes in an open subset $\Omega_{0}$ of $\Omega$, then $u$ must also vanish in the $W$ extension of $\Omega_{0}$ in $\Omega$ consisting of all points of $\Omega$ which can be connected to points of $\Omega_{0}$ by polygonal paths in $\Omega$ with sides defining vectors in $W$.

We consider next the problem of characterizing geometrically the open sets in $R_{n}$ which are $P$-convex with respect to a differential operator $P(D)$ with constant coefficients. An open set $\Omega$ in $R_{n}$ is called $P$-convex if to every compact subset $K_{1}$ of $\Omega$ there exists a compact subset $K_{2}$ of $\Omega$ such that for every $u \in \mathscr{E}^{\prime}(\Omega)$ (distribution with compact support in $\Omega$ ),

$$
\operatorname{supp} P(-D) u \subset K_{1} \quad \text { implies } \quad \operatorname{supp} u \subset K_{2} .
$$

The importance of this concept lies in the following property of $P$-convex sets

Presented to the Society, January 25, 1969 under the title Convexity with respect to differential operators with flat characteristic cones; received by the editors June 28, 1968.

( $\left.{ }^{1}\right)$ This work was sponsored by the National Science Foundation Grant GP 07422. 
proved by Malgrange [2]: The equation $P(D) u=f$ in $\Omega$ has a distribution (or a $C^{\infty}$ ) solution $u$ for every $f$ in $C^{\infty}(\Omega)$ if and only if $\Omega$ is $P$-convex.

Obviously an open set $\Omega$ is $P$-convex if and only if every connected component of $\Omega$ is $P$-convex. It is well known (see [1] or [3]) that an open connected set $\Omega$ is $P$-convex with respect to every differential operator $P$ if and only if $\Omega$ is convex. Furthermore, every open set $\Omega$ is $P$-convex if and only if $P$ is elliptic. For a general differential operator some sufficient and some necessary conditions for $P$-convexity are also known (see [1] and [3]). However, a complete characterization of $P$-convex sets is only known when $n=2$ : An open connected set $\Omega$ in $R_{2}$ is $P$-convex if and only if every characteristic line intersects $\Omega$ in an open interval.

In $\S 3$ we show that if the characteristic cone of $P(D)$ lies in the orthogonal complement of a subspace $W$ of $R_{n}$ different from $\{0\}$ and if $K$ is any compact subset of an open set $\Omega$ in $R_{n}$ then for every $u \in \mathscr{E}^{\prime}(\Omega)$

$$
\text { supp } P(D) u \subset K \text { implies } \operatorname{supp} u \subset \hat{K}(W, \Omega)
$$

where $\hat{K}(W, \Omega)$ is the $W$-hull of $K$ in $\Omega$ consisting of $K$ and of the components of intersections of translations of $W$ with the complement $\mathscr{C} K$ of $K$ which are relatively compact in $\Omega$. This result immediately yields the following sufficient condition for $P$-convexity: $\Omega$ is $P$-convex if for every compact subset $K$ of $\Omega, \hat{K}(W, \Omega)$ is compact. In $\S 4$ we show that for first order differential operators this condition is also necessary for $P$-convexity.

The author wishes to express his sincere thanks to Professor Francois Treves of Purdue University for his constant advice, criticism and encouragement of this work.

2. An application of Holmgren's theorem. Holmgren's theorem asserts the uniqueness of the classical solution of the Cauchy problem when the initial surface is not characteristic with respect to the differential operator. A $C^{1}$ surface is said to be characteristic at a point with respect to $P(x, D)$ if its normal belongs to the characteristic cone of $P(x, D)$ at that point. Hormander [1] has extended Holmgren's theorem to distribution solutions.

LEMma 1 (HoRMANDER). In an open set $\Omega$ of $R_{n}$ let $P(x, D)$ be a differential operator with analytic coefficients and assume that the hyperplanes $x_{n}=$ constant are nowhere characteristic with respect to $P(x, D)$ in $\Omega$. If $u$ is a distribution in $\Omega$ such that $P(x, D) u=0$ in $\Omega_{c}=\left\{x: x \in \Omega, x_{n}<c\right\}$ and if $\Omega_{c} \cap$ supp $u$ is relatively compact in $\Omega$, then $u=0$ in $\Omega_{c}$.

We need a slightly modified form of this lemma which is easily obtained by an analytic transformation of coordinates.

LEMMA 2. In an open set $\Omega$ of $R_{n}$ let $P(x, D)$ be a differential operator with analytic coefficients. Let $F(x)$ be a real-valued analytic function defined in $R_{n}$, having the form

$$
F(x)=f\left(x^{\prime}\right)-x_{n}, \quad x^{\prime}=\left(x_{1}, \ldots, x_{n-1}\right),
$$


and suppose that the level surfaces of $F$ are nowhere characteristic with respect to $P(x, D)$ in $\Omega$. If $u$ is a distribution in $\Omega$ such that $P(x, D) u=0$ in $\Omega_{c}=\{x: x \in \Omega$, $F(x)>c\}$ for some $c$ and if $\Omega_{c} \cap \operatorname{supp} u$ is relatively compact in $\Omega$, then $u=0$ in $\Omega_{c}$.

Definition 1. Let $\Omega$ be an open set in $R_{n}, \Omega_{0}$ an open subset of $\Omega$ and $W$ a subspace of $R_{n}$ different from $\{0\}$. The $W$-extension of $\Omega_{0}$ in $\Omega$, denoted by $\widetilde{\Omega}_{0}(W, \Omega)$ is the union of $\Omega_{0}$ and the set of points of $\Omega$ which can be connected to points of $\Omega_{0}$ by polygonal paths in $\Omega$ with sides defining vectors in $W$.

Clearly $\tilde{\Omega}_{0}(W, \Omega)$ is an open subset of $\Omega$. Moreover, it is easy to see that the following is an equivalent definition:

$\tilde{\Omega}_{0}(W, \Omega)$ is the union of all connected components of intersections of translations of $W$ with $\Omega$ which intersect $\Omega_{0}$.

THEOREM 1. Let $P(x, D)$ be a differential operator with analytic coefficients in an open set $\Omega$ and suppose that at each point $x \in \Omega$ the characteristic cone of $P(x, D)$ is contained in the orthogonal complement of a fixed subspace $W$ of $R_{n}$ different from $\{0\}$. Then every distribution $u$ in $\Omega$ satisfying the equation $P(x, D) u=0$ in $\Omega$ and vanishing in an open subset $\Omega_{0}$ of $\Omega$ must also vanish in $\tilde{\Omega}_{0}(W, \Omega)$.

Explicitly the hypothesis of the theorem means that at every point $x \in \Omega$,

$$
P_{m}(x, \xi)=0 \text { implies } \xi \in W^{\perp} .
$$

Proof of Theorem 1. We consider first the special case in which $W$ is a onedimensional subspace of $R_{n}$. If $W$ is generated by the vector $l$, the hypothesis of the theorem implies that at every point $x \in \Omega$,

$$
\langle\xi, l\rangle \neq 0 \Rightarrow P_{m}(x, \xi) \neq 0 .
$$

By a rotation of coordinates we may assume that $l=(0, \ldots, 0,1)$.

Now let $x^{1} \in \tilde{\Omega}_{0}(W, \Omega)$. We will show that any distribution $u$ in $\Omega$, satisfying $P(x, D) u=0$ in $\Omega$ and vanishing in $\Omega_{0}$, must also vanish in a neighborhood of $x^{1}$. By definition of $\tilde{\Omega}_{0}(W, \Omega)$, there is a point $x^{0} \in \Omega_{0}$ such that the straight line segment $x^{0} x^{1}$ is contained in $\Omega$ and is parallel to the $x_{n}$-axis. Thus

$$
x^{1}=\left(x^{\prime 0}, x_{n}^{1}\right), \quad x^{0}=\left(x^{\prime 0}, x_{n}^{0}\right),
$$

and we may assume without loss of generality that $x_{n}^{1}>x_{n}^{0}$. Clearly there are constants $\varepsilon>0$ and $r>0$ such that the cylinder

$$
U=\left\{x:\left|x^{\prime}-x^{\prime 0}\right|<r, x_{n}^{0}<x_{n}<x_{n}^{1}+\varepsilon\right\}
$$

is contained in $\Omega$ and its base

$$
\left\{x:\left|x^{\prime}-x^{\prime 0}\right|<r, x_{n}=x_{n}^{0}\right\}
$$

is contained in $\Omega_{0}$. Consider now the function

$$
F(x)=\left(x_{n}^{1}+\varepsilon\right)-\left(x_{n}^{1}+\varepsilon-x_{n}^{0}\right)\left|x^{\prime}-x^{\prime 0}\right|^{2} / r^{2}-x_{n}
$$


which is defined and analytic in $R_{n}$. The level surfaces of $F$ are paraboloids with common axis parallel to the $x_{n}$-axis. The normals to these surfaces always have nonzero components in the direction of the $x_{n}$-axis. Hence the hypothesis implies that the level surfaces of $F$ are nowhere characteristic with respect to $P(x, D)$ in $U$. Moreover, if we set

$$
U_{\varepsilon / 2}=\{x: x \in U, F(x)>\varepsilon / 2\},
$$

then $U_{\varepsilon / 2} \cap \operatorname{supp} u$ is relatively compact in $U$. It follows from Lemma 2 that $u=0$ in $U_{\varepsilon^{\prime} / 2}$ which is an open neighborhood of $x^{1}$.

In the general case in which $W$ is a nonzero subspace of $R_{n}$, the hypothesis of the theorem implies that at each point $x \in \Omega$,

$$
\xi \notin W^{\perp} \Rightarrow P_{m}(x, \xi) \neq 0 .
$$

The method of proof in this case consists of a repeated application of the method of proof of the above special case: Let $x^{1}$ be a point of $\Omega$ which can be joined to a point $x^{0} \in \Omega_{0}$ by a straight line segment contained in $\Omega$ and such that the vector $x^{0} x^{1} \in W$. The paraboloidal surfaces with common axis the line passing through $x^{0}$ and $x^{1}$ (of the type constructed in the proof of the special case) are nowhere characteristic with respect to $P(x, D)$ in $\Omega$. This follows from the hypothesis, and from the fact that the normals to these surfaces have nonzero components in the direction of $x^{0} x^{1} \in W$. It follows, as in the proof of the special case, that $u=0$ in an open neighborhood of the segment $x^{0} x^{1}$. Now let $x^{2}$ be a point in $\Omega$ such that the straight line segment $x^{1} x^{2}$ is contained in $\Omega$ and the vector $x^{1} x^{2} \in W$. Since $u=0$ in an open neighborhood of $x^{1}$ we can again conclude that $u=0$ in an open neighborhood of the segment $x^{1} x^{2}$. It should be clear now that repeating this argument a finite number of times completes the proof of the theorem.

It should be noted that the use of paraboloidal surfaces in proving uniqueness theorems for the Cauchy problem is standard. See for example the paper by F. John $[4, \S 2]$.

EXAMPLE 1. If $P(x, D)$ is elliptic in $\Omega$, then $W=R_{n}$ and Theorem 1 states the well-known fact that any solution of a homogeneous elliptic equation in $\Omega$ which vanishes in an open subset $\Omega_{0}$ must also vanish in all connected components of $\Omega$ which contain pieces of $\Omega_{0}$.

EXAmple 2. Consider the heat operator

$$
P(D)=D_{1}^{2}+\cdots+D_{n-1}^{2}-D_{n}
$$

in an open set $\Omega$ of $R_{n}$. Here $W$ is the (n-1)-dimensional subspace of $R_{n}$ containing the $x_{1}, \ldots, x_{n-1}$ axes. Theorem 1 states that if $u$ is a solution of $P(D) u=0$ in $\Omega$ and $u=0$ in the open subset $\Omega_{0}$ of $\Omega$, then $u$ must also vanish in $\widetilde{\Omega}_{0}(W, \Omega)$ which in this case consists of all points of $\Omega$ which can be connected to points of $\Omega_{0}$ by polygonal paths in $\Omega$ which lie in hyperplanes normal to the $x_{n}$-axis (characteristic hyperplanes). 
EXAMPLE 3. Consider the first order differential operator in an open set $\Omega$ of $R_{n}$,

$$
P(x, D)=\sum_{j=1}^{n} a^{j} D_{j}+c(x)
$$

where $a^{j}, j=1, \ldots, n$, are (complex valued) constants and $c(x)$ is analytic in $\Omega$. In this case, $W$ is the subspace of $R_{n}$ generated by $\operatorname{Re} a$ and $\operatorname{Im} a$. If $u$ is a solution of $P(x, D) u=0$ in $\Omega \subset R_{n}$ and if $u=0$ in the open subset $\Omega_{0}$ of $\Omega$ then $u=0$ in $\widetilde{\Omega}_{0}(W, \Omega)$ which in this case consists of all points of $\Omega$ which can be connected to points of $\Omega_{0}$ by polygonal paths in $\Omega$ with sides defining vectors which are linear combinations of $\operatorname{Re} a$ and $\operatorname{Im} a$.

\section{A sufficient condition for $P$-convexity.}

Definition 3. Let $\Omega$ be an open set in $R_{n}, K$ a compact subset of $\Omega$ and $W$ a subspace of $R_{n}$ different from $\{0\}$. The $W$-hull of $K$ in $\Omega$, denoted by $\hat{K}(W, \Omega)$, is the union of $K$ and the set of points of $\mathscr{C} K$ which cannot be connected to points of $\mathscr{C} \Omega$ or to infinity by polygonal paths in $\mathscr{C} K$ with sides defining vectors in $W$.

An equivalent definition is the following: $\hat{K}(W, \Omega)$ is the union of $K$ and all components of intersections of translations of $W$ with $\mathscr{C} K$ which are relatively compact subsets of $\Omega$.

THEOREM 2. Let $P(x, D)$ be a differential operator with analytic coefficients in an open neighborhood of the closure of an open set $\Omega$ in $R_{n}$ and suppose that at each point of this neighborhood the characteristic cone of $P(x, D)$ is contained in the orthogonal complement of a fixed subspace $W$ of $R_{n}$ different from $\{0\}$. Let $K$ be a compact subset of $\Omega$. Then for every $u \in \mathscr{E}^{\prime}(\Omega)$,

$$
\text { supp } P(x, D) u \subset K \text { implies } \operatorname{supp} u \subset \hat{K}(W, \Omega) \text {. }
$$

Proof. The complement of $\hat{K}(W, \Omega)$ consists of all components of intersections of translations of $W$ with $\mathscr{C} K$ which are either unbounded or intersect the complement of $\Omega$. Such components certainly intersect the complement of every compact subset $L$ of $\Omega$ containing $K$. It follows that the complement of $\hat{K}(W, \Omega)$ is contained in the $W$-extension of $\mathscr{C} L$ in $\mathscr{C} K$ for every compact subset $L$ of $\Omega$ containing $K$. Let us denote this extension by $U_{L}$. Now for every $u \in \mathscr{E}^{\prime}(\Omega)$ there is a compact subset $L$ of $\Omega$ such that $u=0$ in $\mathscr{C} L$. If, in addition, $P(x, D) u=0$ in $\mathscr{C} K$, it follows from Theorem 1 that $u=0$ in $U_{L}$. We have shown that every $u \in \mathscr{E}^{\prime}(\Omega)$ such that supp $P(x, D) u \subset K$ vanishes in an open set $U_{L}$ containing the complement of $\hat{K}(W, \Omega)$. The proof is complete.

For elliptic differential operators, Theorem 2 yields the following well-known result:

COROLlaRY 1. Under the conditions of Theorem 2 and if $W=R_{n}$, i.e. if $P(x, D)$ is elliptic, the set $\hat{K}(W, \Omega)$ is the compact subset of $\Omega$ consisting of the union of $K$ and all connected components of $\mathscr{C} K$ which are contained in $\Omega$. 
Since $\hat{K}(W, \Omega)$ is always a bounded set contained in $\Omega$, Theorem 2 yields immediately a sufficient condition for $P$-convexity.

Theorem 3. Let $P(D)$ be a differential operator in $R_{n}$ with constant coefficients and suppose that the characteristic cone of $P(D)$ is contained in the orthogonal complement of a subspace $W$ of $R_{n}$ different from $\{0\}$. An open set $\Omega$ in $R_{n}$ is $P$-convex if for every compact subset $K$ of $\Omega, \hat{K}(W, \Omega)$ is compact.

Corollary 2. Every open set $\Omega$ in $R_{n}$ is P-convex with respect to every elliptic differential operator with constant coefficients.

For differential operators of the first order we can show that the sufficient condition for $P$-convexity of Theorem 3 is also necessary.

\section{Characterization of $P$-convex sets for first-order differential operators.}

Lemma 3. Let $\Omega$ be an open set in $R_{n}$ and $W$ a subspace of $R_{n}$ different from $\{0\}$. Suppose that for some compact subset $K$ of $\Omega$ the set $\hat{K}(W, \Omega)$ is not compact. Then there is a sequence of components of intersections of translations of $W$ with $\mathscr{C} K$ which are relatively compact in $\Omega$ such that their distance from $\mathscr{C} \Omega$ tends to zero.

Proof. Since $\hat{K}(W, \Omega)$ is not compact, there is a sequence $\left\{x^{(k)}\right\}$ of points in $\hat{K}(W, \Omega)$ converging to a point $x^{0}$ belonging to the complement of $\hat{K}(W, \Omega)$. Since $K$ is compact, we may assume that $\left\{x^{(k)}\right\}$ is not contained in $K$. Let $\tau W_{k}^{\prime}$ denote the component of the intersection of the translation of $W$ with $\mathscr{C} K$ which is relatively compact in $\Omega$ and which contains the point $x^{(k)}$. Let $\tau W_{0}^{\prime}$ denote the component of the intersection of the translation of $W$ with $\mathscr{C} K$ which contains $x^{0}$. Since $x^{0}$ belongs to the complement of $\hat{K}(W, \Omega), \tau W_{0}^{\prime}$ must be either unbounded or it must intersect $\mathscr{C} \Omega$. It is sufficient to show that the first possibility cannot occur. If $\tau W_{0}^{\prime}$ is unbounded, $x^{0}$ is the end point of an unbounded polygonal path which lies in $\tau W_{0}^{\prime}$. This path is closed and does not intersect $K$, hence its distance from $K$ is positive. This clearly implies that for sufficiently large $k, \tau W_{k}^{\prime}$ are also unbounded, a contradiction.

THEOREM 4. In $R_{n}$ consider the first-order differential operator with constant coefficients

$$
P(D)=\sum_{j=1}^{n} a^{j} D_{j}+c
$$

and let $W$ be the subspace of $R_{n}$ generated by $\operatorname{Re} a$ and $\operatorname{Im} a$. An open set $\Omega$ in $R_{n}$ is $P$-convex if and only if for every compact subset $K$ of $\Omega, \hat{K}(W, \Omega)$ is compact.

Proof. In view of Theorem 3, since the characteristic cone of $P(D)$ is the orthogonal complement of $W$, we need only to prove the necessity. Let $K$ be a compact subset of $\Omega$ such that $\hat{K}(W, \Omega)$ is not compact. We must show that $\Omega$ is not $P$-convex. According to Lemma 3 , there is a sequence $\left\{\tau W_{k}^{\prime}\right\}$ of components of 
intersections and translations of $W$ with $\mathscr{C} K$ which are relatively compact in $\Omega$ such that their distance from $\mathscr{C} \Omega$ tends to zero. We note that for each $k$, the boundary of $\tau W_{k}^{\prime}$ lies in the compact subset $K$ of $\Omega$. The proof will be complete if we can show that for each $k$ there is a distribution $u_{k} \in \mathscr{E}^{\prime}(\Omega)$ such that supp $u_{k}$ is the closure of $\tau W_{k}^{\prime}$ and supp $P(-D) u_{k}$ is the boundary of $\tau W_{k}^{\prime}$. For simplicity, let us assume that $\operatorname{Re} a$ and $\operatorname{Im} a$ are linearly independent, so that $W$ is two-dimensional. An obvious modification is necessary if $\operatorname{Re} a$ and $\operatorname{Im} a$ are linearly dependent. Let $\tau W_{k}$ denote the translation of $W$ containing $\tau W_{k}^{\prime}$. Introduce new coordinates $\left(x_{1}^{\prime}, x_{2}^{\prime}, \ldots, x_{n}^{\prime}\right)$ with origin in $\tau W_{k}$ such that $x_{1}^{\prime}$ and $x_{2}^{\prime}$ are coordinates for $\tau W_{k}$ and set

$$
v_{k}\left(x^{\prime}\right)=1\left(x_{1}^{\prime}, x_{2}^{\prime}\right) \otimes \delta\left(x_{3}^{\prime}\right) \otimes \cdots \otimes \delta\left(x_{n}^{\prime}\right)
$$

Clearly $\operatorname{supp} v_{k}=\tau W_{k}$ and

$$
\sum_{j=1}^{n} a^{j} D_{j} v_{k}=0
$$

Now let $w$ be any analytic solution of

$$
-\sum_{j=1}^{n} a^{j} D_{j} w+c=0 \quad \text { in } R_{n}
$$

and set

$$
\tilde{u}_{k}=e^{w} v_{k} \text {. }
$$

It is easy to verify that $P(-D) \tilde{u}_{k}=0$ and supp $\tilde{u}_{k}=\tau W_{k}$. Finally, set

$$
u_{k}=\chi\left(\tau W_{k}^{\prime}\right) \tilde{u}_{k}
$$

where $\chi\left(\tau W_{k}^{\prime}\right)$ is the characteristic function of $\tau W_{k}^{\prime}$. Clearly $u_{k}$ has the required properties.

\section{BIBLIOGRAPHY}

1. L. Hormander, Linear partial differential operators, Academic Press, New York, 1963.

2. B. Malgrange, Existence et approximation des solutions des équations aux dérivées partielles et des équations de convolution, Ann. Inst. Fourier Grenoble 6 (1955-56), 271-355.

3. F. Treves, Linear partial differential equations with constant coefficients, Gordon and Breach, New York, 1966.

4. F. John, On linear partial differential equations with analytic coefficients, Comm. Pure Appl. Math. 2 (1949), 209-253.

Purdue University,

LAFAYETTE, INDIANA 